\section{Crystal structure of 1,1'-bis(2-methoxy- carbonyl-2-methylpropyl)ferrocene}

\author{
Yan-Feng Guo, ${ }^{\mathrm{a}}$ Jian-Jun Wang, ${ }^{\mathrm{a}}$ Wei-Juan $\mathrm{Xu},{ }^{\mathrm{a}}$ Dong-Hao \\ Sun ${ }^{\mathrm{a}}$ and Qiang $\mathrm{Gao}^{\mathrm{b} *}$
}

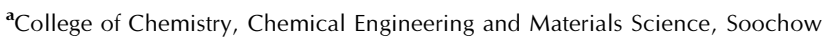
University, Suzhou, Jiangsu 215123, People's Republic of China, and ${ }^{\mathbf{b}}$ Key Laboratory of Eco-textiles, Ministry of Education, College of Textile \& Clothing, Jiangnan University, Wuxi 214122, People's Republic of China. *Correspondence e-mail: gaoqiang@jiangnan.edu.cn

Received 26 October 2015; accepted 31 October 2015

Edited by M. Weil, Vienna University of Technology, Austria

The Fe atom in the title ferrocene derivative, $\left[\mathrm{Fe}\left(\mathrm{C}_{11} \mathrm{H}_{15} \mathrm{O}_{2}\right)_{2}\right]$, is situated on an inversion centre. As a result of the pointgroup symmetry $\overline{1}$ of the molecule, the ferrocene moiety adopts a staggered conformation. The average $\mathrm{Fe}-\mathrm{C}(\mathrm{Cp})$ bond length ( $\mathrm{Cp}$ is cyclopentadienyl) is 2.045 (4) $\AA$, in agreement with that of other disubstituted ferrocenes. The $\mathrm{Fe}-\mathrm{C}$ bond length involving the substituted $\mathrm{C}$ atom is slightly longer [2.0521 (17) $\AA]$ than the remaining $\mathrm{Fe}-\mathrm{C}$ bond lengths caused by the inductive effect of the methylene group on the $\mathrm{Cp}$ ring. Apart from van der Waals forces, no significant intermolecular interactions are observed in the crystal packing.

Keywords: crystal structure; inversion symmetry; disubstituted ferrocene; ester.

CCDC reference: 1434467

\section{Related literature}

The interest in disubstituted ferrocene compounds has increased due to their applications in the field of homogeneous catalysis, biology and medicine (Atkinson et al., 2004; Gao et al., 2009; Ferreira et al., 2006). The presence of ester groups on these compounds make them promising candidates for the construction of metal-containing polymers (Wilbert $e t$ al., 1995). Related structures have been described by Woodward et al. (1952); Cetina et al. (2003); Navarro et al. (2004); Pérez et al. (2015).

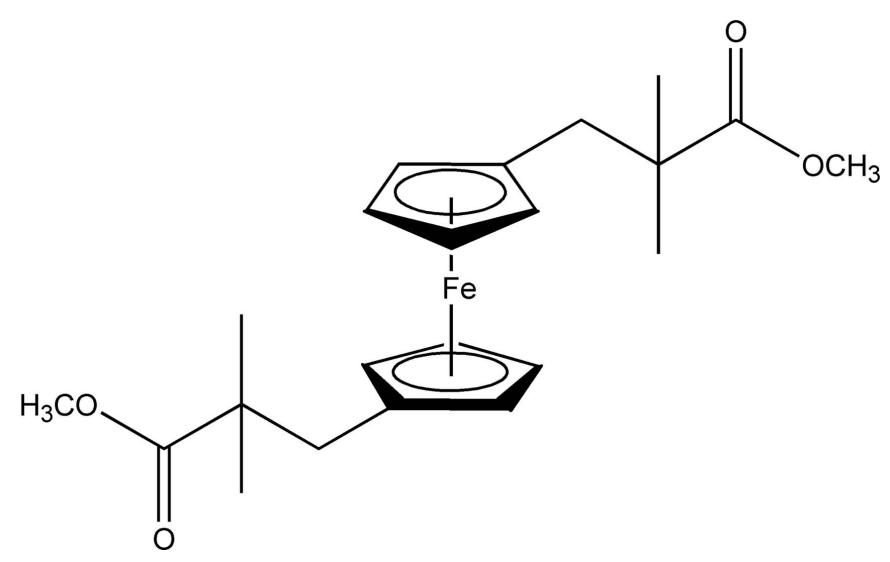

\section{Experimental}

\subsection{Crystal data}

$\left[\mathrm{Fe}\left(\mathrm{C}_{11} \mathrm{H}_{15} \mathrm{O}_{2}\right)_{2}\right]$

$M_{r}=414.31$

Triclinic, $P \overline{1}$

$a=6.273(3) \AA$

$b=8.313$ (4) $\AA$

$c=10.490(5) \AA$

$\alpha=83.833(6)^{\circ}$

$\beta=74.405(7)^{\circ}$

$$
\begin{aligned}
& \gamma=81.652(8)^{\circ} \\
& V=520.0(4) \AA^{3} \\
& Z=1 \\
& \text { Mo } K \alpha \text { radiation } \\
& \mu=0.75 \mathrm{~mm}^{-1} \\
& T=296 \mathrm{~K} \\
& 0.15 \times 0.12 \times 0.12 \mathrm{~mm}
\end{aligned}
$$

\subsection{Data collection}

Bruker APEXII CCD

diffractometer

Absorption correction: multi-scan (SADABS; Krause et al., 2015)

$T_{\min }=0.896, T_{\max }=0.916$

2753 measured reflections 1793 independent reflections 1688 reflections with $I>2 \sigma(I)$ $R_{\text {int }}=0.013$

\subsection{Refinement}

$R\left[F^{2}>2 \sigma\left(F^{2}\right)\right]=0.029$

$w R\left(F^{2}\right)=0.074$

$S=1.05$

1793 reflections

\author{
124 parameters \\ $\mathrm{H}$-atom parameters constrained \\ $\Delta \rho_{\max }=0.21{\mathrm{e} \AA^{-3}}^{-3}$ \\ $\Delta \rho_{\min }=-0.17 \mathrm{e}^{-3}$
}

Data collection: APEX2 (Bruker, 2012); cell refinement: SAINT (Bruker, 2012); data reduction: $S A I N T$; $\operatorname{program}(\mathrm{s})$ used to solve structure: SHELXS97 (Sheldrick, 2008); program(s) used to refine structure: SHELXL97 (Sheldrick, 2008); molecular graphics: DIAMOND (Brandenburg \& Berndt, 1999); software used to prepare material for publication: SHELXTL (Sheldrick, 2008).

\title{
Acknowledgements
}

We are very grateful for the financial support from the Open Project Program of Key Laboratory of Eco-textiles, Ministry of Education, Jiangnan University (No. KLET1303).

Supporting information for this paper is available from the IUCr electronic archives (Reference: WM5231). 


\section{References}

Atkinson, R. C. J., Gibson, V. C. \& Long, N. J. (2004). Chem. Soc. Rev. 33, 313 328.

Brandenburg, K. \& Berndt, M. (1999). DIAMOND. University of Bonn, Germany.

Bruker (2012). APEX2 and SAINT. Bruker AXS Inc., Madison, Wisconsin, USA.

Cetina, M., Jukić, M., Rapić, V. \& Golobič, A. (2003). Acta Cryst. C59, m212m214.

Ferreira, C. L., Ewart, C. B., Barta, C. A., Little, S., Yardley, V., Martins, C., Polishchuk, E., Smith, P. J., Moss, J. R., Merkel, M., Adam, M. J. \& Orvig, C. (2006). Inorg. Chem. 45, 8414-8422.
Gao, L. M., Hernández, R., Matta, J. \& Meléndez, E. (2009). Metal-based Drugs, Article ID 420784, doi: 10.1155/2009/420784.

Krause, L., Herbst-Irmer, R., Sheldrick, G. M. \& Stalke, D. (2015). J. Appl. Cryst. 48, 3-10.

Navarro, A.-E., Spinelli, N., Moustrou, C., Chaix, C., Mandrand, B. \& Brisset, H. (2004). Nucleic Acids Res. 32, 5310-5319.

Pérez, W. I., Rheingold, A. L. \& Meléndez, E. (2015). Acta Cryst. E71, 536539.

Sheldrick, G. M. (2008). Acta Cryst. A64, 112-122.

Wilbert, G., Wiesemann, A. \& Zentel, R. (1995). Macromol. Chem. Phys. 196, 3771-3788.

Woodward, R. B., Rosenblum, M. \& Whiting, M. C. (1952). J. Am. Chem. Soc. 74, 3458-3459. 


\title{
supporting information
}

Acta Cryst. (2015). E71, m213-m214 [https://doi.org/10.1107/S2056989015020642]

\section{Crystal structure of 1,1'-bis(2-methoxycarbonyl-2-methylpropyl)ferrocene}

\author{
Yan-Feng Guo, Jian-Jun Wang, Wei-Juan Xu, Dong-Hao Sun and Qiang Gao
}

\section{S1. Experimental}

1,1'-bis(1-methoxy-methyl)ferrocene was first prepared by refluxing 1,1'-bis(hydroxymethyl)ferrocene in methanol and acetic acid $(12: 1 v / v)$ for $16 \mathrm{~h}$. Then a solution of 1,1,-bis(1-methoxy-methyl)ferrocene (3.481 g, $12.7 \mathrm{mmol})$, 1-methoxy-1-(trimethylsiloxy)-2-methyl-1-propene (10.5 ml, $50.8 \mathrm{mmol})$ and $\mathrm{BF}_{3}-\mathrm{OEt}_{2}(3.5 \mathrm{ml}, 27.9 \mathrm{mmol})$ in $\mathrm{CH}_{2} \mathrm{Cl}_{2}(180$ $\mathrm{ml}$ ) was stirred at $195 \mathrm{~K}$ for $15 \mathrm{~min}$. The reaction was quenched with a satured solution of $\mathrm{NaHCO}_{3}$ and extracted with $\mathrm{CH}_{2} \mathrm{Cl}_{2}$. The organic phases were combined and dried to give a viscous yellow oil, which was chromatographed over a column of silica gel using ethyl acetate/petroleum ether $(1: 4 v / v)$ as the eluent. Yellow crystals of the title compound were obtained by slow evaporation of a solution in dichloromethane/petroleum ether (333-363 K). ${ }^{1} \mathrm{H} \mathrm{NMR}\left(400 \mathrm{MHz}, \mathrm{CDCl}_{3}\right)$ $\delta 3.97\left(\mathrm{~d}, 8 \mathrm{H}, \mathrm{C}_{5} \mathrm{H}_{4} \mathrm{FeC}_{5} \mathrm{H}_{4}\right), 3.62\left(\mathrm{~s}, 6 \mathrm{H}, \mathrm{OCH}_{3}\right), 2.57$ (s, $\left.4 \mathrm{H}, \mathrm{CH}_{2}\right), 1.08$ (s, 12H, $\left.\mathrm{C}\left(\mathrm{CH}_{3}\right)_{2}\right)$. HRMS (ESI): $\mathrm{C}_{22} \mathrm{H}_{30} \mathrm{FeO}_{4}$ calcd for $[M+\mathrm{H}]^{+} 415.1572$, found 415.1575 .

\section{S2. Refinement}

$\mathrm{H}$ atoms were placed in calculated positions and thereafter treated as riding atoms, with $\mathrm{C}-\mathrm{H}=0.98 \AA$ and $U_{\text {iso }}(\mathrm{H})=$ $1.2 U_{\mathrm{eq}}(\mathrm{C})(\mathrm{Cp}$ rings $\mathrm{CH}), 0.97 \AA$ and $U_{\mathrm{iso}}(\mathrm{H})=1.2 U_{\mathrm{eq}}(\mathrm{C})\left(\right.$ methylene $\left.\mathrm{CH}_{2}\right)$ and $0.96 \AA U_{\text {iso }}(\mathrm{H})=1.5 U_{\text {eq }}(\mathrm{C})\left(\right.$ methyl $\left.\mathrm{CH}_{3}\right)$.

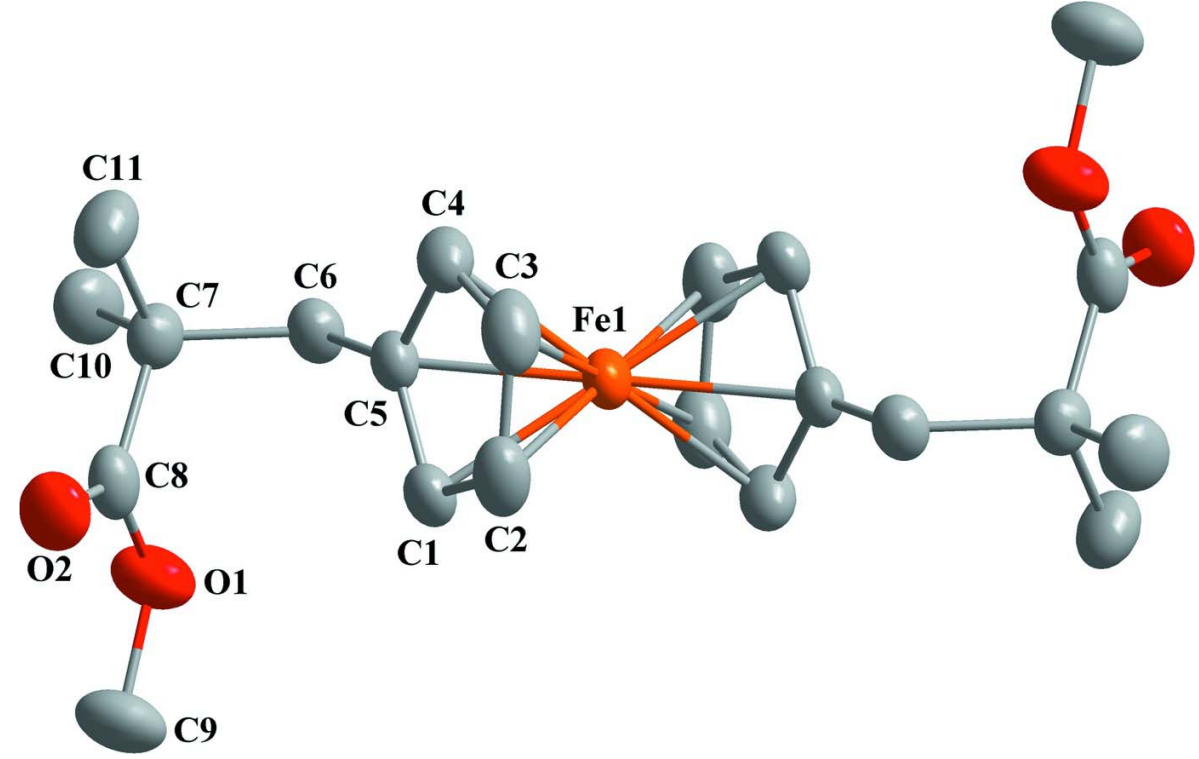

Figure 1

The molecular structure of the title complex, showing displacement ellipsoids drawn at the $50 \%$ probability level. All $\mathrm{H}$ atoms have been omitted for clarity. Unlabelled atoms are related to labelled ones by the symmetry operation $-x,-y+1,-z$ +1 . 


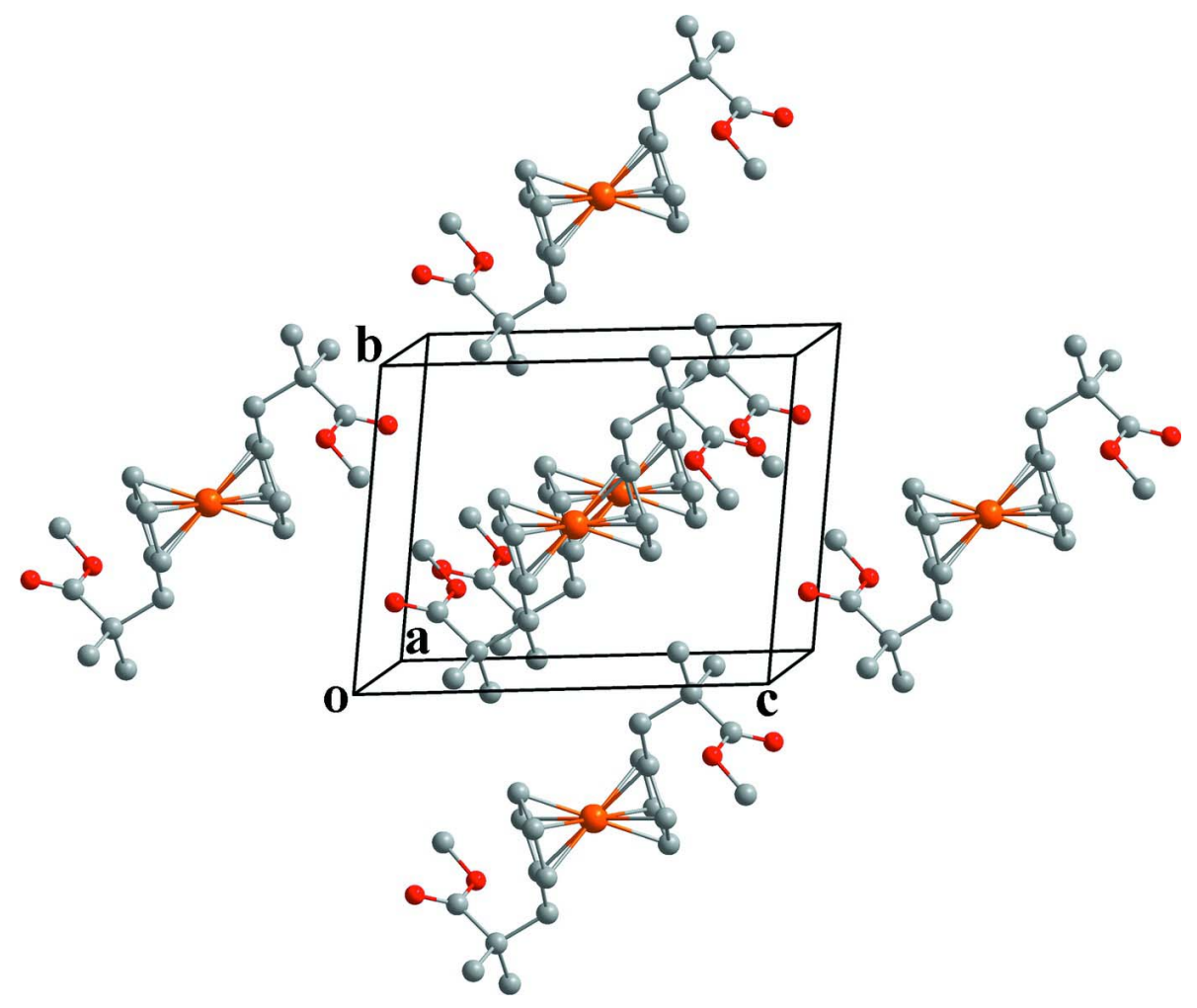

Figure 2

The packing of molecules in the crystal structure of the title compound.

1,1'-Bis(2-methoxycarbonyl-2-methylpropyl)ferrocene

\section{Crystal data}

$\left[\mathrm{Fe}\left(\mathrm{C}_{11} \mathrm{H}_{15} \mathrm{O}_{2}\right)_{2}\right]$

$M_{r}=414.31$

Triclinic, $P \overline{1}$

Hall symbol: -P 1

$a=6.273(3) \AA$

$b=8.313(4) \AA$

$c=10.490(5) \AA$

$\alpha=83.833(6)^{\circ}$

$\beta=74.405(7)^{\circ}$

$\gamma=81.652(8)^{\circ}$

$V=520.0(4) \AA^{3}$

Data collection

\section{Bruker APEXII CCD}

diffractometer

Radiation source: fine-focus sealed tube

Graphite monochromator

$\varphi$ and $\omega$ scans

Absorption correction: multi-scan

(SADABS; Krause et al., 2015)

$T_{\min }=0.896, T_{\max }=0.916$
$Z=1$

$F(000)=220$

$D_{\mathrm{x}}=1.323 \mathrm{Mg} \mathrm{m}^{-3}$

Mo $K \alpha$ radiation, $\lambda=0.71073 \AA$

Cell parameters from 1589 reflections

$\theta=3.3-28.2^{\circ}$

$\mu=0.75 \mathrm{~mm}^{-1}$

$T=296 \mathrm{~K}$

Block, yellow

$0.15 \times 0.12 \times 0.12 \mathrm{~mm}$

2753 measured reflections

1793 independent reflections

1688 reflections with $I>2 \sigma(I)$

$R_{\text {int }}=0.013$

$\theta_{\text {max }}=25.0^{\circ}, \theta_{\text {min }}=2.0^{\circ}$

$h=-7 \rightarrow 5$

$k=-9 \rightarrow 9$

$l=-12 \rightarrow 12$ 


\section{Refinement}

Refinement on $F^{2}$

Least-squares matrix: full

$R\left[F^{2}>2 \sigma\left(F^{2}\right)\right]=0.029$

$w R\left(F^{2}\right)=0.074$

$S=1.05$

1793 reflections

124 parameters

0 restraints

Primary atom site location: structure-invariant direct methods
Secondary atom site location: difference Fourier map

Hydrogen site location: inferred from neighbouring sites

$\mathrm{H}$-atom parameters constrained

$w=1 /\left[\sigma^{2}\left(F_{\mathrm{o}}^{2}\right)+(0.038 P)^{2}+0.1517 P\right]$ where $P=\left(F_{\mathrm{o}}{ }^{2}+2 F_{\mathrm{c}}{ }^{2}\right) / 3$

$(\Delta / \sigma)_{\max }=0.001$

$\Delta \rho_{\max }=0.21 \mathrm{e} \AA^{-3}$

$\Delta \rho_{\min }=-0.17$ e $\AA^{-3}$

Special details

Geometry. All e.s.d.'s (except the e.s.d. in the dihedral angle between two l.s. planes) are estimated using the full covariance matrix. The cell e.s.d.'s are taken into account individually in the estimation of e.s.d.'s in distances, angles and torsion angles; correlations between e.s.d.'s in cell parameters are only used when they are defined by crystal symmetry. An approximate (isotropic) treatment of cell e.s.d.'s is used for estimating e.s.d.'s involving 1.s. planes.

Refinement. Refinement of $F^{2}$ against ALL reflections. The weighted $R$-factor $w R$ and goodness of fit $S$ are based on $F^{2}$, conventional $R$-factors $R$ are based on $F$, with $F$ set to zero for negative $F^{2}$. The threshold expression of $F^{2}>\sigma\left(F^{2}\right)$ is used only for calculating $R$-factors(gt) etc. and is not relevant to the choice of reflections for refinement. $R$-factors based on $F^{2}$ are statistically about twice as large as those based on $F$, and $R$ - factors based on ALL data will be even larger.

Fractional atomic coordinates and isotropic or equivalent isotropic displacement parameters $\left(\AA^{2}\right)$

\begin{tabular}{lllll}
\hline & $x$ & $y$ & $z$ & $U_{\text {iso }} / U_{\text {eq }}$ \\
\hline Fe1 & 0.0000 & 0.5000 & 0.5000 & $0.03254(14)$ \\
O1 & $0.6653(2)$ & $0.24987(19)$ & $0.15534(14)$ & $0.0536(4)$ \\
O2 & $0.4288(3)$ & $0.2353(2)$ & $0.03404(15)$ & $0.0646(4)$ \\
C8 & $0.4892(3)$ & $0.1969(2)$ & $0.13268(18)$ & $0.0397(4)$ \\
C5 & $0.1330(3)$ & $0.3282(2)$ & $0.36443(16)$ & $0.0335(4)$ \\
C1 & $0.1796(3)$ & $0.4864(2)$ & $0.30665(17)$ & $0.0387(4)$ \\
H1A & 0.3282 & 0.5203 & 0.2685 & $0.046^{*}$ \\
C6 & $0.2998(3)$ & $0.1817(2)$ & $0.37491(17)$ & $0.0377(4)$ \\
H6A & 0.2343 & 0.1094 & 0.4496 & $0.045^{*}$ \\
H6B & 0.4288 & 0.2169 & 0.3931 & $0.045^{*}$ \\
C4 & $-0.1038(3)$ & $0.3335(2)$ & $0.40734(18)$ & $0.0410(4)$ \\
H4A & -0.1861 & 0.2426 & 0.4513 & $0.049^{*}$ \\
C7 & $0.3785(3)$ & $0.0848(2)$ & $0.24845(18)$ & $0.0372(4)$ \\
C11 & $0.1817(4)$ & $0.0227(3)$ & $0.2186(2)$ & $0.0547(6)$ \\
H11A & 0.2329 & -0.0363 & 0.1400 & $0.082^{*}$ \\
H11B & 0.0746 & 0.1134 & 0.2049 & $0.082^{*}$ \\
H11C & 0.1134 & -0.0482 & 0.2920 & $0.082^{*}$ \\
C10 & $0.5508(4)$ & $-0.0594(3)$ & $0.2709(2)$ & $0.0519(5)$ \\
H10A & 0.6012 & -0.1197 & 0.1930 & $0.078^{*}$ \\
H10B & 0.4836 & -0.1293 & 0.3452 & $0.078^{*}$ \\
H10C & 0.6753 & -0.0192 & 0.2884 & $0.078^{*}$ \\
C2 & $-0.0263(4)$ & $0.5867(3)$ & $0.31440(18)$ & $0.0467(5)$ \\
H2A & -0.0442 & 0.7018 & 0.2825 & $0.056^{*}$ \\
C9 & $0.7892(5)$ & $0.3576(3)$ & $0.0540(3)$ & $0.0721(7)$ \\
H9A & 0.9106 & 0.3869 & 0.0826 & $0.108^{*}$ \\
& & & &
\end{tabular}




\begin{tabular}{lllll} 
H9B & 0.6925 & 0.4542 & 0.0387 & $0.108^{*}$ \\
H9C & 0.8468 & 0.3033 & -0.0267 & $0.108^{*}$ \\
C3 & $-0.1995(4)$ & $0.4931(3)$ & $0.3759(2)$ & $0.0488(5)$ \\
H3A & -0.3593 & 0.5316 & 0.3944 & $0.059^{*}$ \\
\hline
\end{tabular}

Atomic displacement parameters $\left(\AA^{2}\right)$

\begin{tabular}{lllllll}
\hline & $U^{11}$ & $U^{22}$ & $U^{33}$ & $U^{12}$ & $U^{13}$ & $U^{23}$ \\
\hline Fe1 & $0.0326(2)$ & $0.0351(2)$ & $0.0286(2)$ & $-0.00167(15)$ & $-0.00463(15)$ & $-0.00833(14)$ \\
O1 & $0.0475(8)$ & $0.0685(10)$ & $0.0448(8)$ & $-0.0180(7)$ & $-0.0123(7)$ & $0.0122(7)$ \\
O2 & $0.0833(12)$ & $0.0734(11)$ & $0.0426(8)$ & $-0.0069(9)$ & $-0.0298(8)$ & $0.0029(8)$ \\
C8 & $0.0437(11)$ & $0.0398(10)$ & $0.0332(10)$ & $0.0076(8)$ & $-0.0094(8)$ & $-0.0109(8)$ \\
C5 & $0.0359(9)$ & $0.0366(9)$ & $0.0269(8)$ & $-0.0039(7)$ & $-0.0040(7)$ & $-0.0089(7)$ \\
C1 & $0.0447(11)$ & $0.0408(10)$ & $0.0270(9)$ & $-0.0049(8)$ & $-0.0019(8)$ & $-0.0060(7)$ \\
C6 & $0.0409(10)$ & $0.0396(10)$ & $0.0306(9)$ & $-0.0009(8)$ & $-0.0075(8)$ & $-0.0034(7)$ \\
C4 & $0.0371(10)$ & $0.0479(11)$ & $0.0395(10)$ & $-0.0079(8)$ & $-0.0069(8)$ & $-0.0138(8)$ \\
C7 & $0.0375(10)$ & $0.0338(9)$ & $0.0394(10)$ & $0.0018(8)$ & $-0.0098(8)$ & $-0.0073(8)$ \\
C11 & $0.0513(12)$ & $0.0519(13)$ & $0.0651(14)$ & $-0.0067(10)$ & $-0.0150(11)$ & $-0.0224(11)$ \\
C10 & $0.0533(13)$ & $0.0396(11)$ & $0.0557(12)$ & $0.0082(9)$ & $-0.0100(10)$ & $-0.0017(9)$ \\
C2 & $0.0610(13)$ & $0.0430(11)$ & $0.0338(10)$ & $0.0073(10)$ & $-0.0139(9)$ & $-0.0068(8)$ \\
C9 & $0.0694(16)$ & $0.0802(18)$ & $0.0595(15)$ & $-0.0259(14)$ & $-0.0050(13)$ & $0.0195(13)$ \\
C3 & $0.0414(11)$ & $0.0622(13)$ & $0.0449(11)$ & $0.0074(10)$ & $-0.0155(9)$ & $-0.0199(10)$ \\
& & & & & &
\end{tabular}

Geometric parameters $\left(\AA,{ }^{\circ}\right)$

\begin{tabular}{|c|c|c|c|}
\hline $\mathrm{Fe} 1-\mathrm{C} 2^{\mathrm{i}}$ & $2.043(2)$ & $\mathrm{C} 6-\mathrm{C} 7$ & $1.554(3)$ \\
\hline $\mathrm{Fe} 1-\mathrm{C} 2$ & $2.043(2)$ & C6-H6A & 0.9700 \\
\hline $\mathrm{Fe} 1-\mathrm{C} 4^{\mathrm{i}}$ & $2.044(2)$ & C6-H6B & 0.9700 \\
\hline $\mathrm{Fe} 1-\mathrm{C} 4$ & $2.044(2)$ & $\mathrm{C} 4-\mathrm{C} 3$ & $1.418(3)$ \\
\hline $\mathrm{Fe} 1-\mathrm{C} 3^{\mathrm{i}}$ & $2.044(2)$ & $\mathrm{C} 4-\mathrm{H} 4 \mathrm{~A}$ & 0.9800 \\
\hline $\mathrm{Fe} 1-\mathrm{C} 3$ & $2.044(2)$ & $\mathrm{C} 7-\mathrm{C} 11$ & $1.522(3)$ \\
\hline $\mathrm{Fe} 1-\mathrm{C}^{\mathrm{i}}$ & 2.0445 (19) & $\mathrm{C} 7-\mathrm{C} 10$ & $1.536(3)$ \\
\hline $\mathrm{Fe} 1-\mathrm{C} 1$ & $2.0445(19)$ & $\mathrm{C} 11-\mathrm{H} 11 \mathrm{~A}$ & 0.9600 \\
\hline $\mathrm{Fe} 1-\mathrm{C}^{\mathrm{i}}$ & $2.0521(17)$ & C11-H11B & 0.9600 \\
\hline $\mathrm{Fe} 1-\mathrm{C} 5$ & $2.0521(17)$ & $\mathrm{C} 11-\mathrm{H} 11 \mathrm{C}$ & 0.9600 \\
\hline $\mathrm{O} 1-\mathrm{C} 8$ & $1.333(3)$ & $\mathrm{C} 10-\mathrm{H} 10 \mathrm{~A}$ & 0.9600 \\
\hline $\mathrm{O} 1-\mathrm{C} 9$ & $1.446(3)$ & $\mathrm{C} 10-\mathrm{H} 10 \mathrm{~B}$ & 0.9600 \\
\hline $\mathrm{O} 2-\mathrm{C} 8$ & $1.192(2)$ & $\mathrm{C} 10-\mathrm{H} 10 \mathrm{C}$ & 0.9600 \\
\hline $\mathrm{C} 8-\mathrm{C} 7$ & $1.522(3)$ & $\mathrm{C} 2-\mathrm{C} 3$ & $1.400(3)$ \\
\hline $\mathrm{C} 5-\mathrm{C} 1$ & $1.424(3)$ & $\mathrm{C} 2-\mathrm{H} 2 \mathrm{~A}$ & 0.9800 \\
\hline $\mathrm{C} 5-\mathrm{C} 4$ & $1.428(3)$ & C9-H9A & 0.9600 \\
\hline $\mathrm{C} 5-\mathrm{C} 6$ & $1.501(3)$ & C9-H9B & 0.9600 \\
\hline $\mathrm{C} 1-\mathrm{C} 2$ & $1.420(3)$ & $\mathrm{C} 9-\mathrm{H} 9 \mathrm{C}$ & 0.9600 \\
\hline $\mathrm{C} 1-\mathrm{H} 1 \mathrm{~A}$ & 0.9800 & $\mathrm{C} 3-\mathrm{H} 3 \mathrm{~A}$ & 0.9800 \\
\hline $\mathrm{C} 2 \mathrm{i}-\mathrm{Fe} 1-\mathrm{C} 2$ & 180.0 & $\mathrm{C} 2-\mathrm{C} 1-\mathrm{C} 5$ & $108.24(18)$ \\
\hline $\mathrm{C} 2^{\mathrm{i}}-\mathrm{Fe} 1-\mathrm{C} 4^{\mathrm{i}}$ & $67.98(9)$ & $\mathrm{C} 2-\mathrm{C} 1-\mathrm{Fe} 1$ & $69.61(10)$ \\
\hline $\mathrm{C} 2-\mathrm{Fe} 1-\mathrm{C} 4^{\mathrm{i}}$ & $112.02(9)$ & $\mathrm{C} 5-\mathrm{C} 1-\mathrm{Fe} 1$ & $69.95(10)$ \\
\hline
\end{tabular}




\begin{tabular}{|c|c|}
\hline $\mathrm{C} 22^{\mathrm{i}}-\mathrm{Fe} 1-\mathrm{C} 4$ & $112.02(9)$ \\
\hline $\mathrm{C} 2-\mathrm{Fe} 1-\mathrm{C} 4$ & $67.98(9)$ \\
\hline $\mathrm{C} 4-\mathrm{Fe} 1-\mathrm{C} 4$ & 180.0 \\
\hline $\mathrm{C} 2^{\mathrm{i}}-\mathrm{Fe} 1-\mathrm{C} 3^{\mathrm{i}}$ & $40.08(9)$ \\
\hline $\mathrm{C} 2-\mathrm{Fe} 1-\mathrm{C}^{\mathrm{i}}$ & $139.92(9)$ \\
\hline $\mathrm{C} 4^{\mathrm{i}}-\mathrm{Fe} 1-\mathrm{C} 3^{\mathrm{i}}$ & $40.61(8)$ \\
\hline $\mathrm{C} 4-\mathrm{Fe} 1-\mathrm{C}^{\mathrm{i}}$ & $139.39(8)$ \\
\hline $\mathrm{C} 22^{\mathrm{i}}-\mathrm{Fe} 1-\mathrm{C} 3$ & $139.92(9)$ \\
\hline $\mathrm{C} 2-\mathrm{Fe} 1-\mathrm{C} 3$ & $40.08(9)$ \\
\hline $\mathrm{C} 4-\mathrm{Fe} 1-\mathrm{C} 3$ & $139.39(8)$ \\
\hline $\mathrm{C} 4-\mathrm{Fe} 1-\mathrm{C} 3$ & $40.61(8)$ \\
\hline $\mathrm{C} 3{ }^{\mathrm{i}}-\mathrm{Fe} 1-\mathrm{C} 3$ & 180.0 \\
\hline $\mathrm{C} 2^{\mathrm{i}}-\mathrm{Fe} 1-\mathrm{C} 1^{\mathrm{i}}$ & $40.65(8)$ \\
\hline $\mathrm{C} 2-\mathrm{Fe} 1-\mathrm{C} 1^{\mathrm{i}}$ & $139.35(8)$ \\
\hline $\mathrm{C} 4^{\mathrm{i}}-\mathrm{Fe} 1-\mathrm{C}^{\mathrm{i}}$ & $68.18(8)$ \\
\hline $\mathrm{C} 4-\mathrm{Fe} 1-\mathrm{Cl}^{\mathrm{i}}$ & $111.82(8)$ \\
\hline $\mathrm{C} 3^{\mathrm{i}}-\mathrm{Fe} 1-\mathrm{C} 1^{\mathrm{i}}$ & $68.00(9)$ \\
\hline $\mathrm{C} 3-\mathrm{Fe} 1-\mathrm{C}^{\mathrm{i}}$ & $112.00(9)$ \\
\hline $\mathrm{C} 2 \mathrm{i}-\mathrm{Fe} 1-\mathrm{C} 1$ & $139.35(8)$ \\
\hline $\mathrm{C} 2-\mathrm{Fe} 1-\mathrm{C} 1$ & $40.65(8)$ \\
\hline $\mathrm{C} 4-\mathrm{Fe} 1-\mathrm{C} 1$ & $111.82(8)$ \\
\hline $\mathrm{C} 4-\mathrm{Fe} 1-\mathrm{C} 1$ & $68.18(8)$ \\
\hline 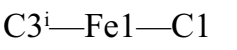 & $112.00(9)$ \\
\hline $\mathrm{C} 3-\mathrm{Fe} 1-\mathrm{C} 1$ & $68.00(9)$ \\
\hline $\mathrm{C} 1{ }^{\mathrm{i}}-\mathrm{Fe} 1-\mathrm{C} 1$ & 180.0 \\
\hline $\mathrm{C} 2^{\mathrm{i}}-\mathrm{Fe} 1-\mathrm{C} 5^{\mathrm{i}}$ & $68.48(8)$ \\
\hline $\mathrm{C} 2-\mathrm{Fe} 1-\mathrm{C} 5^{\mathrm{i}}$ & $111.52(8)$ \\
\hline $\mathrm{C} 4^{\mathrm{i}}-\mathrm{Fe} 1-\mathrm{C} 5^{\mathrm{i}}$ & $40.80(8)$ \\
\hline $\mathrm{C} 4-\mathrm{Fe} 1-\mathrm{C} 5^{\mathrm{i}}$ & $139.20(8)$ \\
\hline $\mathrm{C} 3^{\mathrm{i}}-\mathrm{Fe} 1-\mathrm{C} 5^{\mathrm{i}}$ & $68.51(8)$ \\
\hline $\mathrm{C} 3-\mathrm{Fe} 1-\mathrm{C} 5^{\mathrm{i}}$ & $111.49(8)$ \\
\hline $\mathrm{C} 1^{\mathrm{i}}-\mathrm{Fe} 1-\mathrm{C} 5^{\mathrm{i}}$ & $40.67(7)$ \\
\hline $\mathrm{C} 1-\mathrm{Fe} 1-\mathrm{C} 5^{\mathrm{i}}$ & $139.33(7)$ \\
\hline $\mathrm{C} 22^{\mathrm{i}}-\mathrm{Fe} 1-\mathrm{C} 5$ & $111.52(8)$ \\
\hline $\mathrm{C} 2-\mathrm{Fe} 1-\mathrm{C} 5$ & $68.48(8)$ \\
\hline $\mathrm{C} 4-\mathrm{Fe} 1-\mathrm{C} 5$ & $139.20(8)$ \\
\hline $\mathrm{C} 4-\mathrm{Fe} 1-\mathrm{C} 5$ & $40.80(8)$ \\
\hline $\mathrm{C} 33^{\mathrm{i}-\mathrm{Fe} 1-\mathrm{C} 5}$ & $111.49(8)$ \\
\hline $\mathrm{C} 3-\mathrm{Fe} 1-\mathrm{C} 5$ & $68.51(8)$ \\
\hline $\mathrm{C} 1{ }^{\mathrm{i}}-\mathrm{Fe} 1-\mathrm{C} 5$ & $139.33(7)$ \\
\hline $\mathrm{C} 1-\mathrm{Fe} 1-\mathrm{C} 5$ & $40.67(7)$ \\
\hline $\mathrm{C} 5^{\mathrm{i}}-\mathrm{Fe} 1-\mathrm{C} 5$ & $180.00(7)$ \\
\hline $\mathrm{C} 8-\mathrm{O} 1-\mathrm{C} 9$ & $117.84(18)$ \\
\hline $\mathrm{O} 2-\mathrm{C} 8-\mathrm{O} 1$ & $123.17(19)$ \\
\hline $\mathrm{O} 2-\mathrm{C} 8-\mathrm{C} 7$ & $125.6(2)$ \\
\hline $\mathrm{O} 1-\mathrm{C} 8-\mathrm{C} 7$ & $111.19(16)$ \\
\hline $\mathrm{C} 1-\mathrm{C} 5-\mathrm{C} 4$ & $106.96(17)$ \\
\hline $\mathrm{C} 1-\mathrm{C} 5-\mathrm{C} 6$ & $126.84(17)$ \\
\hline
\end{tabular}

$\begin{array}{ll}\text { C2-C1-H1A } & 125.9 \\ \text { C5-C1-H1A } & 125.9 \\ \text { Fe1-C1-H1A } & 125.9 \\ \text { C5-C6-C7 } & 113.97(15) \\ \text { C5-C6-H6A } & 108.8 \\ \text { C7-C6-H6A } & 108.8 \\ \text { C5-C6-H6B } & 108.8 \\ \text { C7-C6-H6B } & 108.8 \\ \text { H6A-C6-H6B } & 107.7 \\ \text { C3-C4-C5 } & 108.21(18) \\ \text { C3-C4-Fe1 } & 69.70(12) \\ \text { C5-C4-Fe1 } & 69.92(10) \\ \text { C3-C4-H4A } & 125.9 \\ \text { C5-C4-H4A } & 125.9 \\ \text { Fe1-C4-H4A } & 125.9 \\ \text { C11-C7-C8 } & 110.12(17) \\ \text { C11-C7-C10 } & 109.95(17) \\ \text { C8-C7-C10 } & 108.85(16) \\ \text { C11-C7-C6 } & 110.52(16) \\ \text { C8-C7-C6 } & 108.53(15) \\ \text { C10-C7-C6 } & 108.84(16) \\ \text { C7-C11-H11A } & 109.5 \\ \text { C7-C11-H11B } & 109.5 \\ \text { H11A-C11-H11B } & 109.5 \\ \text { C7-C11-H11C } & 109.5 \\ \text { H11A-C11-H11C } & 109.5 \\ \text { H11B-C11-H11C } & 109.5 \\ \text { C7-C10-H10A } & 109.5 \\ \text { C7-C10-H10B } & 109.5 \\ \text { H10A-C10-H10B } & 109.5 \\ \text { C7-C10-H10C } & 109.5 \\ \text { H10A-C10-H10C } & 109.5 \\ \text { H10B-C10-H10C } & 109.5 \\ \text { C3-C2-C1 } & 108.31(18) \\ \text { C3-C2-Fe1 } & 69.99(12) \\ \text { C1-C2-Fe1 } & 69.73(11) \\ \text { C3-C2-H2A } & 125.8 \\ \text { C1-C2-H2A } & 125.8 \\ \text { Fe1-C2-H2A } & 125.8 \\ \text { O1-C9-H9A } & 109.5 \\ \text { O1-C9-H9B } & 109.5 \\ \text { H9A-C9-H9B } & 109.5 \\ \text { O1-C9-H9C } & 109.5 \\ \text { H9A-C9-H9C } & 109.5 \\ \text { H9B-C9-H9C } & \\ \text { C2-C3-C4 } & \\ \text { C2-C3-Fe1 } & \\ \text { C4-C3-Fe1 } & \\ & \end{array}$




$\begin{array}{llll}\mathrm{C} 4-\mathrm{C} 5-\mathrm{C} 6 & 126.17(17) & \mathrm{C} 2-\mathrm{C} 3-\mathrm{H} 3 \mathrm{~A} & 125.9 \\ \mathrm{C} 1-\mathrm{C} 5-\mathrm{Fe} 1 & 69.38(10) & \mathrm{C} 4-\mathrm{C} 3-\mathrm{H} 3 \mathrm{~A} & 125.9 \\ \mathrm{C} 4-\mathrm{C} 5-\mathrm{Fe} 1 & 69.28(10) & \mathrm{Fe} 1-\mathrm{C} 3-\mathrm{H} 3 \mathrm{~A} & 125.9 \\ \mathrm{C} 6-\mathrm{C} 5-\mathrm{Fe} 1 & 127.75(13) & & \end{array}$

Symmetry code: (i) $-x,-y+1,-z+1$. 